\title{
Interactions of Axion Photons in Magnetised Media
}

\author{
Emmanuel B. Umoren, Joseph G. Atat, Augustine A. Umoh, Imeh E. Essien, \\ Cyril C. Umoren and Akaninyene D. Antia \\ Department of Physics, Faculty of Science, University of Uyo, Nigeria.
}

\begin{abstract}
We have compared between the standard model and generational model of particle physics. By means of a special Lagrangian function we consider the interaction of photon axions in both magnetic field and magnetic medium. We also present a polynomial in powers of the external magnetic field with even and odd powers in $B$ and showed that the axion coupling mass $(M)$ assumes two eigenvalues.
\end{abstract}

DOI: $10.7176 /$ APTA/83-01

Publication date: February $29^{\text {th }} 2020$

\subsection{Introduction}

The Standard model (SM) of particle physics describes all the transition probabilities for interactions involving the six leptons and six quarks which form the elementary particles of Generational Model (GM) in terms of only three unified additive quantum number $[1,2,3]$.

The SM describes the strong, weak and electromagnetic interactions among elementary particles. The energy scale that characterizes the symmetry breaking is modeled through the interaction Lagrangian as:

$$
L=f^{-1} J^{\mu} \partial_{\mu} \phi
$$

where, $J^{\mu}$ is the Noether current of the spontaneously broken global symmetry, $f$ is the decay constant and $\phi$ is the light bosons. If $f$ is very large, these particles interact very weakly [4].

Axions are spontaneous breaking of continuous symmetries always generating various excitations with varying mass spectra [5,6]. Axions remains of particular interest because the Peccei-Quinn (PQ) mechanism remains perhaps the most credible scheme to preserve Charge-Parity (CP) in Quantum Chromo-Dynamics (QCD) $[7,8]$. Moreover, the cold dark matter of the universe may well consist of axions.

Axions which appear as Nambu-Goldstone bosons of the spontaneously broken PQ symmetry $U_{P Q}(1)$ are one of the well-motivated candidates for the cold dark matter $[9,10]$. Axions generically interacts with photons according to the Lagrangian:

$$
\begin{aligned}
& L_{a \gamma}=-\frac{g_{a \gamma}}{4} F_{\mu v} \widetilde{F}^{\mu v} a \\
& L_{a \gamma}=g_{a \gamma} E . B a
\end{aligned}
$$

with a strength

$$
g_{a \gamma}=\frac{\alpha}{\pi f_{a}} \xi,
$$

where, $f_{a}$ is the energy scale of the symmetry breaking, $\xi$ is the model-dependent factor of order unity, $F_{\mu v}$ is the electromagnetic field strength tensor, $\widetilde{F}^{\mu v}$ its dual and $a$ is the axion field [11].

In the limit of vanishing small quark masses (Chiral limit), strong interaction Lagrangain has a global $U(2)_{v} \times U(2)_{A}$ symmetry. The symmetry group further breaks spontaneously to produce the hadron multiplex. The vector part of the symmetry breaks to the product of iso-spin and baryon number symmetry given by:

$$
U(2)_{v}=S U(2)_{I} \times U(1)_{B}
$$

In nature, baryon number is seen to be conserved and the mass spectrum of nucleon and pion multiplex indicates that the iso-spin part is also conserved approximately.

Mikheev et al.[12 studied the decay of axion photons in an external electromagnetic field using the photon decay $\gamma-\gamma a$, where $a$ is pseudo-scalar particle associated with PQ symmetry. Hagmann et al.[13] studied the axions and other similar particles; they pointed out the photon regeneration, photon polarization and long range forces. Axion photon mixing in a magnetized media was studied by Ganguly [14].

In this letter, we present an alternative to the SM of particle physics. This alternative model, called the GM describes all the transition probabilities for interactions including the six leptons and six quarks. We also look 
into the axionphoton mixing effect and show how this effect can change polarization angle when passing through a magnetic field and give the eigenvalues of axion coupling mass.

\subsection{Standard Model of Interacting Fundamental Particle}

The SM describes interactions of leptons and the multitude of hadrons (baryons and mesons) as well as the decay modes of the unstable leptons and hadrons.

In SM the elementary particles that are the constituents of matter are assumed to be the six leptons: electron neutrino $\left(v_{e}\right)$, electron $\left(e^{-}\right)$, muon neutrino $\left(v_{\mu}\right)$, muon $\left(\mu^{-}\right)$, tau neutrino $\left(v_{\tau}\right)$ and tau $(\tau)$; and the six quarks: up $(u)$, down $(d)$, charmed $(c)$, strange $(s)$, top $(t)$, and bottom $(b)$, together with their antiparticles [15].

These twelve particles are all spin- $\frac{1}{2}$ particles and falls naturally into three families or generations

(i) $\quad v_{e}, e^{-}, u, d$.

(ii) $v_{\mu}, \mu^{-}, c, s$.

(iii) $\quad v_{\tau}, \tau^{-}, t, b$.

Each generation consist of two leptons with charges $Q=0, Q=-1$ and two quarks with charges $Q=+\frac{2}{3}$, $Q=-\frac{1}{3}$

For each particle additive quantum number $N$, the corresponding antiparticle has the additive quantum number $-N$.

Table 1: SM additive quantum number for leptons and quarks

\begin{tabular}{|c|c|c|c|c|c|c|c|c|c|}
\hline \multirow[t]{2}{*}{ Particle } & \multirow{2}{*}{$\begin{array}{c}\text { Charge } \\
Q\end{array}$} & \multicolumn{3}{|c|}{ Lepton numbers } & \multirow{2}{*}{$\begin{array}{c}\text { Baryon number } \\
A\end{array}$} & \multicolumn{4}{|c|}{ Quarks } \\
\hline & & $L$ & $L_{\mu}$ & $L_{\tau}$ & & $S$ & $C$ & $B$ & $T$ \\
\hline$v_{e}$ & 0 & 1 & 0 & 0 & 0 & 0 & 0 & 0 & 0 \\
\hline$e^{-}$ & -1 & 1 & 0 & 0 & 0 & 0 & 0 & 0 & 0 \\
\hline$v_{u}$ & 0 & 1 & 1 & 0 & 0 & 0 & 0 & 0 & 0 \\
\hline$\mu^{-}$ & -1 & 1 & 1 & 0 & 0 & 0 & 0 & 0 & 0 \\
\hline$v$ & 0 & 1 & 0 & 1 & 0 & 0 & 0 & 0 & 0 \\
\hline$\nu_{\tau}^{-}$ & -1 & 1 & 0 & 1 & 0 & 0 & 0 & 0 & 0 \\
\hline$i$ & $+\frac{2}{3}$ & 0 & 0 & 0 & $\frac{1}{3}$ & 0 & 0 & 0 & 0 \\
\hline$d$ & $-\frac{1}{3}$ & 0 & 0 & 0 & $\frac{1}{3}$ & 0 & 0 & 0 & 0 \\
\hline$c$ & $+\frac{2}{3}$ & 0 & 0 & 0 & $\frac{1}{3}$ & 0 & 1 & 0 & 0 \\
\hline$S$ & $-\frac{1}{3}$ & 0 & 0 & 0 & $\frac{1}{3}$ & -1 & 0 & 0 & 0 \\
\hline$S$ & $+\frac{2}{3}$ & 0 & 0 & 0 & $\frac{1}{3}$ & 0 & 0 & 0 & 1 \\
\hline $\begin{array}{l}t \\
b\end{array}$ & $-\frac{1}{3}$ & 0 & 0 & 0 & $\begin{array}{l}3 \\
\frac{1}{3}\end{array}$ & 0 & 0 & -1 & 0 \\
\hline
\end{tabular}

The classification of the elementary particles in SM is non-unified. The additive quantum number $Q$ and baryon number $A$ are assumed to be conserved in strong, electromagnetic and weak interactions. The lepton numbers $L, L_{\mu}$ and $L_{\tau}$ are not conserved in strong interactions but are strictly conserved in both electromagnetic and weak interactions. The quarks $S, C, B$ and $T$ are strictly conserved only in strong and electromagnetic interactions but can undergo a change of one unit in weak interactions [16, 17], 


\subsection{Generational Model of interacting fundamental Particle}

Generational model (GM) is both simpler and unified because leptons and quarks are assigned the same kind of additive quantum number which are: charge $(Q)$, particle number $(p)$ and quantum number $(g)[18,19]$.

Table 2: GM additive quantum number for leptons and quarks

\begin{tabular}{llllcccc}
\hline Particles & $Q$ & $p$ & $g$ & particles & $Q$ & $p$ & $g$ \\
\hline$v_{e}$ & 0 & -1 & 0 & $u$ & $+\frac{2}{3}$ & $\frac{1}{3}$ & 0 \\
$e^{-}$ & -1 & -1 & 0 & $d$ & $-\frac{1}{3}$ & $\frac{1}{3}$ & 0 \\
$v_{\mu}$ & 0 & -1 & \pm 1 & $c$ & $+\frac{2}{3}$ & $\frac{1}{3}$ & \pm 1 \\
$\mu^{-}$ & -1 & -1 & \pm 1 & $s$ & $-\frac{1}{3}$ & $\frac{1}{3}$ & \pm 1 \\
$v_{\tau}$ & 0 & -1 & $0, \pm 2$ & $t$ & $+\frac{2}{3}$ & $\frac{1}{3}$ & $0, \pm 2$ \\
$\tau^{-}$ & -1 & -1 & $0, \pm 2$ & $b$ & $-\frac{1}{3}$ & $\frac{1}{3}$ & $0, \pm 2$ \\
\hline
\end{tabular}

The classification of elementary particle in GM is unified and simple; the corresponding antiparticles have the opposite sign for each particle additive quantum number. These are significant to describe all the observed transition amplitudes for both hadronic and leptonicprocesses provided each force particle mediating the various interactions have $p=g=0$.

\subsection{Comparison of SM and GM}

The SM and GM has only one additive quantum number in common, electric charge $Q$ which serves the role in each model and is conserved. The particle quantum number $p$ replaces both lepton number $L$ and baryon number $A$ of the SM. The generational quantum number $g$ effectively replaces $L_{\mu}, L_{\tau}$ and $S, C, B, T$.

\subsection{Photon Axion in a Magnetic Medium}

The model-dependent factors for the PQ charges for different generation of quarks and leptons [20] is

$$
L_{a f}=\frac{g_{a f}^{\prime}}{m_{f}} \sum_{f}\left(\psi_{f} \gamma_{\mu} \gamma_{5} \psi_{f}\right) \partial^{\mu} a
$$

$g_{a f}^{\prime}=\left(X_{f} m_{f} / f_{a}\right)$ is the Yukawa coupling constant, $m_{f}$ is the fermion mass and $\sum_{f}$ is the sum over all the fermions from each family.

The axion photon mixing Lagrangian in an external magnetic field is:

$$
L_{a \gamma}=-g_{a \gamma \gamma} \frac{e^{2}}{32 \pi^{2}} a F \widetilde{F}^{E X T}
$$

The axion coupling constant is described by:

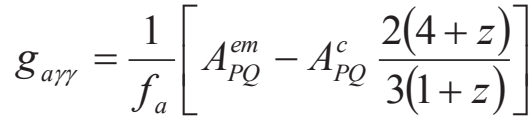

where

$$
z=\frac{m_{u}}{m_{d}}
$$

where, $m_{u}$ and $m_{d}$ are the mass of light quarks. Anomality factors are given by the following relation:

$$
\begin{aligned}
& A_{P Q}^{e m}=\operatorname{Tr}\left(Q_{f}^{2}\right) X_{f} \\
& \delta_{a b} A_{P Q}^{c}=\operatorname{Tr}\left(\lambda_{a} \lambda_{b} X_{f}\right)
\end{aligned}
$$

The trace $(T r)$ is over the fermion species. To estimate the loop induced $\gamma-a$ coupling, we introduce $p^{\prime}=p+k$ so that the effective vertex for the $\gamma-a$ turns out to be: 


$$
i \Gamma_{v}(K)=g_{a f} e Q_{f} \int \frac{d^{4} p}{(2 \pi)^{4}} K^{\mu} \operatorname{Tr}\left[\gamma_{\mu} \gamma_{5} i S(p) \gamma_{v} i S\left(p^{\prime}\right)\right]
$$

where, $S(p)$ is the in-medium fermionic propagator in the external magnetic field, computed to all orders in the field strength. $\mathrm{Eq}(11)$ has the following structure:

$$
\Gamma_{v}(K)=K^{\mu} \Pi_{\mu v}^{A}(K)
$$

Where, $\Pi_{\mu \nu}^{A}$ is the axial polarization tensor which have contributions from magnetic field and magnetic medium. Putting Eq(12) into Eq(11)we have:

$$
i \Pi_{\mu \nu}^{A}(K)=g_{a f} e Q_{f} \int \frac{d^{4} p}{(2 \pi)^{4}} \operatorname{Tr}\left[\gamma_{\mu} \gamma_{5} i S(p) \gamma_{v} i S\left(p^{\prime}\right)\right]
$$

The contribution from magnetic field and magnetic medium effects is given by:

$$
i \Pi_{\mu \nu}^{A}(K)=g_{a f} e Q_{f} \int \frac{d^{4} p}{(2 \pi)^{4}} \operatorname{Tr}\left[\begin{array}{l}
\gamma_{\mu} \gamma_{5} i S_{B}^{v}(p) \gamma_{v} i S_{B}^{v}\left(p^{\prime}\right)+ \\
\gamma_{\mu} \gamma_{5} S_{B}^{\eta}(p) \gamma_{v} i S_{B}^{v}\left(p^{\prime}\right)+ \\
\gamma_{\mu} \gamma_{5} i S_{B}^{v}(p) \gamma_{v} S_{B}^{\eta}\left(p^{\prime}\right)
\end{array}\right]
$$

The pure magnetic field contribution has been estimated by C. Schubert [21].

\subsection{Contribution from Magnetized Medium}

The fermion propagator in external magnetic field and magnetic medium is given by:

$$
\Pi_{\mu v}^{A}(K)=\left(i g_{a f} e Q_{f}\right) \int \frac{d^{4} p}{(2 \pi)^{4}} \int_{-\infty}^{\infty} d s e^{\phi(p, s)} \int_{0}^{\infty} d s^{\prime} e^{\phi\left(p^{\prime}, s^{\prime}\right)} J
$$

where

$$
J=\operatorname{Tr}\left\{\begin{array}{l}
{\left[\gamma_{\mu} \gamma_{5} G(p, s) \gamma_{v} G\left(p^{\prime}, s^{\prime}\right)\right] \eta F(p)+} \\
{\left[\gamma_{\mu} \gamma_{5} G\left(-p^{\prime}, s^{\prime}\right) \gamma_{\nu} G(-p, s)\right] \eta F(-p)}
\end{array}\right\}
$$

Let $J=R_{\mu v}\left(p, p^{\prime}, s, s^{\prime}\right)$ be a polynomial in powers of the external magnetic field with even and odd power of B. Then:

$$
R_{\mu \nu}\left(p, p^{\prime}, s, s^{\prime}\right)=R_{\mu \nu}^{(E)}\left(p, p^{\prime}, s, s^{\prime}\right)+R_{\mu \nu}^{(o)}\left(p, p^{\prime}, s, s^{\prime}\right)
$$

In addition of being even and odd in powers of $e Q_{f} B$, they are also odd and even in powers of chemical potential. Therefore, under charge conjugation

$$
B \& \mu \leftrightarrow(-\mu) \&(-B)
$$

The parity structures are also different. These properties are very useful while analyzing the structure of axion photon coupling using discrete symmetry arguments to justify the presence or absence of magnetic field and magnetic media.

\subsection{Axion Photon Mixing}

The Lagrangian that describes the axion photon dynamics is given by:

$$
L=-\frac{1}{4} F^{\mu v} F_{\mu v}+\frac{1}{4 M} F^{\mu v} \widetilde{F}_{\mu v}+\frac{1}{2}\left(\partial_{\mu} a \partial^{\mu} a-m_{a}^{2} a^{2}\right)
$$

where, $m_{a}$ is the axion mass. This effective Lagraingian shows the effect of mixing of a spin zero pseudo-scalars with two photons. This system contains the dynamics of three degree of freedom. Hence, we have three equations of motion which can be written in compact form:

$$
\left[\left(w^{2}+\partial_{z}^{2}\right) \mathrm{I}+\mathrm{M}\right]\left(\begin{array}{l}
A_{\perp} \\
A_{\|} \\
a
\end{array}\right)=0
$$


where $\mathrm{I}$ is a $3 \times 3$ identity matrix and $\mathrm{M}$ is short hand notation for the following matrix:

$$
\mathrm{M}=\left(\begin{array}{ccc}
0 & 0 & 0 \\
0 & 0 & i g B w \\
0 & -i g B w & -m_{a}^{2}
\end{array}\right)
$$

$\mathrm{A}_{\perp}(z)$ is the photon gauge field with polarization vector directed along the perpendicular to magnetic field. $\mathrm{A}_{||}(z)$ is the remaining component of photon gauge field with polarization vector and $\mathrm{a}(z)$ is the pseudo-scalar axion field.

In the off diagonal matrix of $\operatorname{Eq}(21)$ given by $\pm i g B w$, the magnetic field is:

$$
B=B^{E X T} \sin (\hat{\alpha})
$$

$B^{E X T}$ is the transverse part of the external magnetic field and $\hat{\alpha}$ is the angle between the wave vector and $B^{E X T}$.

$$
M_{2 \times 2}=\left(\begin{array}{cc}
M_{j} & -i g B w \\
i g B w & m_{a}^{2}+M_{j}
\end{array}\right)
$$

We can solve for the eigenvalues of $\operatorname{Eq}(23)$ from the determinant

$$
\left|\begin{array}{cc}
M_{j} & -i g B w \\
i g B w & m_{a}^{2}+M_{j}
\end{array}\right|=0
$$

$j$ assumes two values and the roots are:

$$
M_{ \pm}=-\frac{m_{a}^{2}}{2} \pm \sqrt{\left(\frac{m_{a}^{2}}{2}\right)^{2}+(g B w)^{2}}
$$

where $M$ is the axion coupling mass.

\subsection{Conclusion}

In this paper, we have compared between the standard model and generational model of fundamental particle physics. Table 1 shows that the classification of the elementary particles in SM is non-unified; the additive quantum number $(Q)$ and baryon number $(A)$ are assumed to be conserved in strong, electromagnetic and weak interactions; the lepton numbers $L, L_{\mu}$ and $L_{\tau}$ are not conserved in strong interactions but are strictly conserved in both electromagnetic and weak interactions. Table 2 shows that the classification of elementary particle in GM is unified and simple as it groups the additive quantum numbers into charge $(Q)$, particle number $(p)$ and quantum number $(g)$; the corresponding antiparticles have the opposite sign for each particle additive quantum number.It is pointed out that charge $(Q)$ serves the same role in each model and it's conserved. We use Lagrangian to present axial polarization tensor which has contributions from magnetic field and magnetic medium. In the framework of magnetic medium, a polynomial in powers of the external magnetic field with even and odd powers of $B$ is pointed out. The axial photon mixing contains dynamics of three degree of freedom; hence, we outline equation of motion in compact form and obtain the eigenvalues of the axion coupling mass.

\section{REFERENCES}

[1] Armstrong, D. S.; Arvienx, J.; Asturyen, R.; Averett, T. \& Bailey, S. L. (2005) Strange-Quark contributions to parity-Violating Asymmetries in the forward G0 Electron-Proton scattering experiment. Physics Review Letter. Vol.95, No.9, 09221(5 pages)

[2] Lyre, H. (2008) Does the Higgs mechanism exist? International Studies in the Philosophy of science. Vol.22, No.2, pp. 119-133

[3] Robson B. A. (2009) The general model and origin of mass. International Journal of Modern Physics E. Vol.18, No.8, pp. 1773-1780.

[4] Baker, C. A.; Doyles, D. D.;Geltenbort, P.; Green, K. \& van der Grinten, M. G. D. (2006) Improved experimental limit on the electric Dipole moment of the neutron. Physics Review Letter.D17, 131801

[5] Morrison A. D. \& Robson B. A. (2009) $2 \pi$ decay of the $K_{L}^{0}$ meson without CP violation. International Journal of Modern Physics E. Vol.8, No.9, pp. 1825-1830

[6] Ruz, J.; Vogel, J. K. \&Pivovaroff, M. J. (2015) Recent Constraints on Axion-photon and Axion-electron 
coupling with the CAST experiment. Physics Procedia.pp153-256.

[7] Peccei, R. D. (1996) QCD Strong CP and Axions. Jornal of Korean Physics Society. Vol.29, pp. 145-199

[8] McLean, E.; Davies, C.T.H.; Lytle, A.T. \&Koponen, J. (2004) High-Precision lattice QCD confronts experiment. Physics Review Letter. Vol.92, No.2, 02001

[9] Peccei, R. D. \& Quinn, H, R. (1977) CP conservation in the presence of Instantons. Physics Review Letter. Vol.38, 1440

[10] Peccei, R. D. \& Quinn, H, R. (1977) Constraints imposed by CP conservation in the presence of Instantons. Physics Review Letter. D38, 1791

[11] Raffelt, G. G. (1990) Astrophysical methods to constrain axions and other novel particles. Physics Report. Vol.198,pp. 808-832

[12] Mikheev, N. V.; Parkhomenko, A. Ya. \&Vassilevskaya, L. A. (1999) Axion decay of a photon in an external magnetic field.Physics Review Letter. Vol.83. 9903401

[13] Hagman, C.; Murayama, H.; Raffelt, G. G.; Rosenbery, L. J.; \& vanBibber, K. (2010) Axions and other similar particles. Physics Review Letter. D104, 041301

[14] Ganguly, A. K. (2006) Axion Photon mixing in magnetized media. Annals of Physics. Vol.321, 1457

[15] Evans, P. W. \& Robson, B. A. (2006) Comparison of quark mixing in the Standard and generation model. International Journal of Modern Physics E. Vol.15, No.3, pp. 617-625

[16] Robson, B. A. (2004) Relation between strong and weak Iso-spin.International Journal of Modern Physics E. Vol.11, No. 6, pp. 999-1018

[17] Veltman, M. (2003) Facts and mysteries in elementary particle Physics. World Science Publishing Company. Singapore

[18] Robson, B. A. (2008) The generational model and the electroweak connection. International Journal of Modern Physics E. Vol.18, No.6, pp. 1015-1030

[19] Datta, A. \&Raychaudhuri, S. (1994) Quark masses and mixing angles in a four-generation model with a naturally heavy neutrino. Physics Review D. 49(9), 4762-4772

[20] Ganguly, A. K.; Jain, P.; \&Mandal, S. (2009) Photon and axion oscillation in a magnetized medium. Physics Review Letter. D79, 115014

[21] Schubert, C. (2000) Vacuum polarization tensors in constant electromagnetic field, Part 2. Nuclear Physics B. Vol.585, pp. 429-450 